\title{
Unsteady Transonic Small Disturbance Theory with Strong Shock Waves
}

\section{G. David Kerlick, David Nixon and William F. Ballhaus, Jr.}

\section{IERAN COPY \\ JUL $2 ? 1990$}

I ANCLEY RESEARCH CENTER

LOBRATYY WASA, HAMPTON, VA 


\section{UNSTEADY TRANSONIC SMALL DISTURBANCE THEORY} WITH STRONG SHOCK WAVES

$$
\text { by }
$$

G. David Kerlick

$$
\text { David Nixon }
$$

Nielsen Engineering \& Research, Inc.

$$
\text { and }
$$

William F. Ballhaus, Jr. NASA Anies Research Center

NEAR TR 230

September 1980

Sponsored by NASA Ames Research Center under Contracts $A 70215 B$ and $A 77455 B$ 


\section{UNSTEADY TRANSONIC SMALL DISTURBANCE THEORY WITH STRONG SHOCK WAVES}

\section{INTRODUCTION}

The most common methods of predicting steady flow aerodynamic characteristics at transonic speeds are either the Transonic Small Disturbance (TSD) theory (ref. 1) or the Full Potential Equation (FPE) theory (ref. 2). The more accurate Euler equations solutions (ref. 3) are expensive to obtain, although for flows with strong shock waves such solutions are essential. The FPE theory is based on the assumption that the flow is isentropic and irrotational and generally has a (numerically) exact treatment of the wing boundary conditions. The TSD theory is an approximation to the FPE theory and thin wing boundary conditions are used in the solution procedure. One of the advantages of the TSD theory is the flexibility in deriving the approximate equation. This flexibility is generally utilized by a choice of a transonic scale parameter. The basic assumptions of isentropy and irrotationality in both these theories are only valid when there are no shock waves in the flow or when any shock waves are weak. The generally accepted definition of a weak shock is when the local Mach number just ahead of the shock is less than 1.3. When both TSD and FPE solutions are compared to the more realistic Euler equation solutions it is found that the agreement is satisfactory provided that the basic restriction to weak shock waves is not violated. The use of thin wing boundary conditions can also introduce errors into the TSD solutions. If the flow has strong shock waves, however, then there is considerable aisagreement between both TSD and FPE solutions and Euler equation solutions. Generally the predicted shock location for the potential theories is much further aft than that for the Euler equation solutions. This is due to the isentropic assumption being invalid in these flows. The causes of the error in the shock location in the steady TSD theory for 
two-dimensional flow have been examined in reference 4 where a correction procedure has been derived that allows the basic equation within the formal accuracy bounds of a small disturbance theory. The basic hypothesis of the theory is that the error in shock location is primarily due to the stronger shock predicted by TSD theory compared to the shock strength of the Euler equations. It is also assumed that if the shock strength is suitably corrected then the shock location should be approximately correct. The technique used in reference 4 makes use of two TSD solutions, with different scaling parameters, and an interpolation scheme derived for discontinuous transonic flows. Examples of flows with strong shocks computed with this method agree satisfactorily with the Euler equation solutions, although the use of the thin airfoil boundary conditions in the TSD theory can give rise to errors near the leading edge.

The aim of the present paper is to extend the basic concept of reference 4 to include unsteady transonic potential flows. The only satisfactory unsteady transonic method available is the low frequency theory of Ballhaus and Goorjian (ref. 5) which numerically integrates the nonlinear low frequency transonic small disturbance equation in a time accurate manner. As in steady flow the results for a small disturbance equation formulation compare satisfactorily with solutions of the Euler equations when any shock waves in the flow are weak. However, the accuracy of the solution diminishes, particularly as regards the shock location and motion, as the shocks become stronger. Again, as in the steady flow, it is assumed that this error is due to the shock strength of the small disturbance solution being larger than the corresponding Euler equation solution and that it is this difference that leads to the wrong shock location.

In reference 4 the correction to the TSD equation is obtained by computing two steady state solutions and then using an interpolation technique to give the required solution. This technique is not really fcasible for unsteady flow since the correction procedure is required for each time step in the 
solution. As there may be 1,000 or more such time steps the technique used in reference 4 would require an inordinate amount of computer time to obtain a solution. Consequently, a different means of implementing the shock strength correction is developed in this paper. The technique involves the addition of higher order terms, which are formally of negligible magnitude, to the low frequency TSD equation. These terms are then chosen such that any shock waves in the flow have strengths approximately equal to the appropriate Rankine-Hugoniot shock strength. Two correcting approaches are investigated in the paper. The first is to derive a correction for the mean steady flow and then simply use this corrected form for oscillatory flows. The second is to derive a correction for both steady and oscillatory parts of the flow. This second development is the most satisfactory and comparisons of the present results with Euler equation results are generally favorable, particularly regarding shock location, although there are some discrepancies in the pressure distribution in the leading edge region.

\section{BASIC SMALI DISTURBANCE FORMULATION}

The low frequency TSD equation for the perturbation velocity potential $\phi(x, y)$ at a free-stream Mach number $M_{\infty}$ can be written in the form (ref. 6)

$$
\left(1-M_{\infty}^{2}\right) \phi_{x x}+\phi_{Y Y}=(\gamma+1) M_{\infty}^{q_{\phi}} \phi_{x x}+\frac{2 M_{\infty}^{2} c}{U_{\infty}} \phi_{x t}
$$

where $\gamma$ is the ratio of specific heats and $q$ is the transonic scaling parameter. The two most commonly used (ref. 6) values of $q$ are 2 (Spreiter scaling) and 1.75 (Krupp scaling). In the transonic limit of $M_{\infty} \rightarrow I$ both scalings are identical. The pressure coefficient $c_{p}(x, y, t)$ is given in the low frequency small disturbance theory by 


$$
c_{p}(x, y, t)=-2 \phi_{x}(x, y, t)
$$

Associated with Equation (1) are the usual tangency and far field boundary conditions. The weak shock jump conditions for Equation (1) are

$$
\llbracket\left(1-M_{\infty}^{2}-(\gamma+1) M_{\infty}^{q_{\phi}}\right) \phi_{x} \rrbracket+\tan \phi_{s} \llbracket \phi_{Y} \rrbracket-\frac{2 M_{\infty}^{2} c \dot{x} s}{U_{\infty}} \llbracket \phi_{x} \rrbracket=0
$$

where $\llbracket$ denotes a jump through a shock wave, $\phi_{\mathbf{s}}$ is the angle that the normal to the shock makes with the $\mathrm{x}$-axis and $x_{S}=\left(\frac{d x_{S}}{d t}\right)$ is the shock speed. For a shock normal to the free stream the shock strength, $\sigma_{T^{\prime}}$ is defined as

$$
\sigma_{T}(t)=C_{p}^{-}(t)-c_{p}^{+}(t)=-2\left[c_{p}^{+}(t)-c_{p}^{*}+\frac{4 M_{\infty}^{2} c \dot{x}_{S}(t)}{(\gamma+1) M_{\infty}^{q_{U}}}\right]
$$

where $c_{p}^{+}, c_{p}^{-}$are the pressure coefficients just ahead of and behind the shock and

$$
C_{p}^{*}=\frac{-2\left(1-M_{\infty}^{2}\right)}{(\gamma+1) M_{\infty}^{q}}
$$

For a harmonic oscillation with frequency $\omega$

$$
\dot{x}_{s}=\left|\delta x_{s}\right| \omega e^{i \omega t}
$$

where $\left|\delta x_{s}\right|$ is the magnitude of the shock motion. Now $\left|\delta x_{s}\right| \sim o\left(\delta_{1}\right)$ where $\delta_{1}$ is the amplitude of the oscillatory motion and generally $\left|\delta_{1}\right| \ll 1$. Since in the low frequency equation, Equation (1), it is implied (ref. 6) that 


$$
k=\frac{u C}{U_{\infty}} \sim o\left(B^{2}\right) \ll 1
$$

it can be seen from Equations (4) and (5) that

$$
\frac{4 M_{\infty}^{2} c \dot{x}_{S}}{(\gamma+1) M_{\infty}^{q} U_{\infty}} \sim o\left(\delta_{1} k\right) \ll c_{p}^{*}
$$

Hence, the shock speed can be neglected in the computation of the oscillatory shock strength. Thus

$$
\sigma_{T} \approx-2\left\{C_{p}^{+}(t)-c_{p}^{*}\right\}
$$

For the following shock jump relations for the Euler equations it is assumed that the low frequency assumption noted above still applies. That is, the shock speed is sufficiently small that the quasi-steady relation holds.

Consider now the quasi-steady Euler equation normal shock jump, $\sigma_{E}(t)$, in terms of $M_{\infty}$ and $C_{p}^{+}(t)$ which is given (ref. 7 ) by

$$
\sigma_{E}(t)=\frac{2 \gamma}{\gamma+I}\left[M_{e}^{2}(t)-1\right]\left[\frac{2}{\gamma M_{\infty}^{2}}+c_{p}^{+}(t)\right]
$$

where the lpstream shock Mach number $M_{e}$ is given by

$$
M_{e}^{2}(t)=\frac{1}{(\gamma-1)}\left\{\left[2+(\gamma-1) M_{\infty}^{2}\right] /\left[\frac{\gamma}{2} M_{\infty}^{2} C_{p}^{+}(t)+1\right]^{\frac{\gamma}{\gamma-1}}-2\right\}
$$

The TSD (wIth $q=2.0$ ) and Euler shock strengths are plotted against $C_{p}^{+}$for $M_{\infty}=0.755$ in figure 1 and it can be seen that as $\left|C_{p}^{+}\right|$increases the discrepancy between $\sigma_{T}$ and $\sigma_{E}$ increases. It 
should be noted here that different transonic scalings not only give a different value of $C_{p}^{*}$ but generally a different value of $\mathrm{C}_{\mathrm{p}}^{+}$. Thus, for different scalings the shock strength may vary considerably.

The basic hypot sis of the present theory, as in reference 4 , is that the error in the shock location in both the steady and unsteady TSD solutions is due primarily to the error in the shock strength as exhibited in figure 1. Solutions of the Euler equations give a different pressure jump across a shock wave than potential theories because of the entropy production due to the shock wave. It is suggested that if the TSD equation is altered, still within its formal accuracy bounds, such that the shock jump approximates the Euler equation shock jump, then the resulting equation is a better compromise in representing the actual flow. The reason for this statement is that by matching the shock jump the new equation implicitly introduces an additional mechanism (formally negligible) that cancels the entropy production and rotationality errors in a potential formulation.

The method introduced in reference 4 to modify the TSD equation is to interpolate two TSD results with different scalings, to obtain the ciesired pressure jump. While this technique is adequate for steady flow corrections it is not really adequate for unsteady flow results since it would require two TSD results at each time step. This would lead to an inordinate amount of computer time. Consequently simpler means of modifying the TSD equation are examined; these are discussed in the next section.

\section{MODIFIED SMALL DISTURBANCE EQUATION}

In an initlal attempt to derive a small disturbance equation that will glve a more realistic shock jump the following equation is used.

$$
\left(a+b \phi_{x}+c \phi_{x}^{2}\right) \phi_{x x}+\phi_{y y}=2 M_{\infty}^{2} \frac{c}{U_{\infty}} \phi_{x t}
$$


If $a=1-M_{\infty}^{2}, b=(\gamma+1) M_{\infty}^{q}$ and $c=0$ Equation (10) is identical to Equation (1).

In the following analysis it is assumed that all shock waves are normal to the free stream. In this case contributions from the $\phi_{y y}$ term to the jump relation can be neglected.

Consider for the morent the more general form of Equation (10)

$$
\left.f\left(\phi_{x}\right)\right\rangle_{x x}+\phi_{y y}-2 M_{\infty}^{2} \frac{c}{U_{\infty}} \phi_{x t}=0
$$

The normal shock Jump relation is

$$
F\left(\theta_{x}^{+}, \sigma\right)-2 M_{\infty}^{2} \frac{c}{U_{\infty}} \dot{x}_{s}=0
$$

where $\phi_{x}^{+}$is the value of $\vartheta_{x}$ just ahead of the shock and $\sigma$ is the shock strength. The function $F\left(\phi_{x}^{+}, \sigma\right)$ is given by

$$
F\left(\phi_{x}^{+}, \sigma\right)=\frac{2}{\sigma} \int_{\phi_{X}^{+}-\frac{\sigma}{2}}^{\phi_{x}^{+}} f\left(\phi_{x}\right) d\left(\phi_{x}\right)
$$

The problem is to pick a suitable form of $f\left(\phi_{x}\right)$, and in the following discussion some conditions that should be satisfied by $f\left(\phi_{x}\right)$ are suggested.

(a) In general the function $f\left(\phi_{x}\right)$ will be nonlinear in $\phi_{X}$ which leads to the possibility of multiple parabolıc points when

$$
f_{x}\left(\phi_{x}\right)=0
$$

It is advisable that at least one of the roots of Equation (14) be $\phi_{\mathrm{x}_{\mathrm{C}}}^{*}$ which is the parabolic point for a conventional (say Krupp) TSD solution. Thus 


$$
f\left(o_{x_{C}}^{*}\right)=0
$$

In order to avold unrealistic multiple parabolic points in the domain of interest it is also desirable that $f\left(\phi_{x}\right)$ be a monotonically decreasing function in some range $\phi_{\mathrm{x}_{\min }} \leq \phi_{\mathrm{x}} \leq \phi_{\mathrm{x}_{\max }}$. Thus

$$
\frac{d f}{d\left(\partial_{x}\right)}<0 \text { for } \phi_{x_{\min }} \leq \phi_{x} \leq \phi_{x_{\max }}
$$

(b) The normal shock strength should be the same as the normal Euler shock strength. Neglecting the shock speed term in Equation (11), as discussed in the previous section, this gives

$$
F\left(o_{X^{\prime}}^{+}, \sigma_{E}\right)=0
$$

where $\sigma_{E}$ is given by Equation (8).

These three conditions, given in Equations (15), (16), and (17), will glve the desired shock strength with no undesirable side effects.

Equations (15), (16), and (17) must be satisfied at each Iterative step or time step in order to get the correct shock jump. While there is little option to satisfyıng each of these equations at all Iterative steps at this preliminary stage of the investigation, it is possible to reduce the amount of computational work required for an unsteady example if a steady state solution is first computed.

It is offen the case that the unsteady pressure distribution is related by a small steady perturbation to a mean state, which itself is close to the steady state result. Hence, if a modified small disturbance equation has been evolved for the steady state case it may be possible to use a simple analytic perturbation of the function $f\left(\phi_{x}\right)$ that will give good accuracy in the 
neighborhood of the steady state result. The additional condition for this idea is given below.

(c) For the shock strength to be approximately correct over a range of values close to the steady state value of $\phi_{\mathrm{x}^{\prime}}^{+} \phi_{\mathrm{x}_{\mathrm{S}}}^{+}$, Equation (17) can be expanded as a Taylor's series to give

$$
F\left(\phi_{X_{S}}^{+}, \sigma_{E_{S}}\right)+\left[\left[\frac{\partial F}{\partial \phi_{X}^{+}}\right)+\frac{\partial F}{\partial \sigma_{E}} \frac{d \sigma_{E}}{d \phi_{X}^{+}}\right]_{S}\left(\phi_{X}^{+}-\phi_{X_{S}}^{+}\right)=0
$$

where the subscript $s$ denotes a value in the steady state.

Now by definition

$$
F\left(o_{x_{s}}^{+}, \sigma_{E_{s}}\right)=0
$$

and hence for Equation (18) to be satisfied for a range of $\left(\phi_{x}^{+}-\phi_{x_{s}}^{+}\right)$

$$
\left\{\frac{\partial F}{\partial \phi_{X}^{+}}+\frac{\partial F}{\partial \phi_{E}} \frac{d \sigma_{E}}{d \phi_{X}^{+}}\right\}_{S}=0
$$

For an unsteady flow therefore there are two options for practical calculations.

Option I. - The function $f\left(\phi_{X}\right)$ can be chosen such that Equations (15)-(17) are satisfied at each iterative and time step.

Option II.- Equations (15), (16), and (19) are satisfied for each iterative step in the steady calculations and Equations (15), (16), and (20) are satisfied at each time step for the oscillatory calculation. 
4. PRELIMINARY IMPLEMENTATION OF THE CORRECTION PROCEDURE

It is possible to derrve functions $f\left(\phi_{X}\right)$ that satisfy all of the conditions given in the preceding section, but in a limited study, such as the present one, it is more convenient to satisfy only certain of these conditions explicitly and to test the resulting function with respect to the other conditions. In the following discussion the set of conditions given in Option II are considered.

The most crucial conditions to satisfy are the shock strength conditions, Equations (19) and (20) since the object of the present excercise is to realize the correct shock strengths. consequently, these conditions are satisfied explicitly. For simplicity in derivation, it is assumed that the modifications to the small disturbance equation will be sufficiently small that Equations (15) and (I6) will be, at least, approximately satisfied. This aspect will be considered with each application of the theory.

The form of $f\left(\phi_{x}\right)$ chosen is that of Equation (10), that is

$$
f\left(\phi_{x}\right)=a+b \phi_{x}+c \phi_{x}^{2}
$$

where $a, b, c$ are either constants of simple functions of $\phi_{x}^{+}$. The particular form of Equations (15), (16), (19), and (20) is now

$$
\begin{aligned}
& a+b \psi_{x}^{*}+c \phi_{x}^{* 2}=0 \\
& \mathrm{~b}+2 \mathrm{c} \dot{\phi}_{\mathrm{x}}<0 \text { for } \phi_{\mathrm{x}_{\text {min }}} \leq \phi_{\mathrm{x}} \leq \phi_{\mathrm{x} \text { max }} \\
& a+b\left(s_{x_{s}}^{+}-\frac{\sigma_{E_{s}}}{2}\right)+c\left({ }_{x_{s}^{+2}}^{+2}-\sigma_{E_{s}} \phi_{x_{s}}^{+}+\frac{\sigma_{E_{s}}^{2}}{3}\right)=0
\end{aligned}
$$




$$
\begin{aligned}
b(1 & \left.-\frac{\sigma E_{s}^{\prime}}{2}\right)+c\left(2 \phi_{x_{S}}^{+}-\sigma_{E_{S}}-\phi_{x_{S}}^{+} \sigma_{E_{S}}^{\prime}+\frac{2}{3} \sigma_{E_{S}} \sigma_{E_{S}}^{\prime}\right) \\
& +\left(\frac{d a}{d \phi_{x}^{+}}\right)_{S}+\left(\phi_{x_{S}}^{+}-\frac{c_{E_{S}}}{2}\right)\left(\frac{d b}{d \phi_{x}^{+}}\right)_{S}+\left(\phi_{x_{S}}^{+2}-\sigma_{E_{S}} \phi_{x_{S}}^{+}+\frac{c_{E_{S}}^{2}}{3}\right)\left(\frac{d c}{d \phi_{x}^{+}}\right)_{S}=0
\end{aligned}
$$

Two applications of this correction were developed as follows.

Method I.- The coefficients $b$ and $c$ were chosen such that for the mean steady case Equation $(24)$ is satisfied with $a=1-M_{\infty}^{2}$ and $b=(\gamma+1) M_{\infty}^{q}$. The coefficients are then fxozen for the unsteady part of the computation. Thus, no further correction is required for the unsteady calculation.

Method II.- The coefficient $\mathrm{c}$ is chosen such that Equation (24) is satisfed and

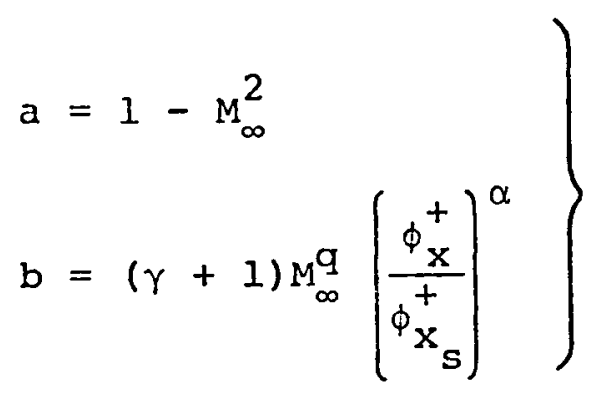

The exponent $\alpha$ is chosen such that equation (25) is satisifed. This form of $f\left(\phi_{X}\right)$ does not have the same propensity for producing multiple parabolic points, as altering $b$ for the steady state solution but incurs the cost of altering $f\left(\phi_{x}\right)$ at each time step by computing ${ }^{+}{ }_{x}$.

In both cases the coefficients $a, b, c$ are chosen such that the shock strength on the upper surface satisfies Equation (20) and these values are retained throughout the flow field. 


\section{NUMERICAI ALGORITHM}

The basic algorithm in the Ballhaus-Goorjian (ref. 5) computer code LTRAN2 solves the equation

$$
\left[F\left(\phi_{X}\right)\right]_{X}+\phi_{Y Y}-2 M_{\infty}^{2} \frac{c}{U_{\infty}} \phi_{x t}=0
$$

in conservation form, where $F\left(\phi_{X}\right)=\left[1-M_{\infty}^{2}-(\gamma+1) M_{\infty}^{q} \phi_{x}\right]$.

The present, modified, form of the TSD equation is in the form of Equation (27) [but with a different $F\left(\phi_{X}\right)$ ] and the same algorithm will suffice. However, because of the type dependent switching of the difference scheme it is important to ensure the conservation properties of the modified TSD solution.

The general form of the type dependent differencing used in LTRAN2, which is applied only to the first term of Equation (27), is

$$
\left.F_{x} \approx \Delta x^{-1}\left[F_{i+\frac{1}{2}}-F_{1-\frac{1}{2}}\right)\left(1-\varepsilon_{i}\right)+\varepsilon_{i-1}\left(F_{i}-\frac{1}{2}-F_{i}-\frac{3}{2}\right)\right]
$$

where $\varepsilon_{i}$ is a swltching operator given by

$$
\varepsilon_{i}=\left\{\begin{array}{l}
0 \text { if } F_{i}>0 \\
1 \text { if } F_{i}<0
\end{array}\right.
$$

The suffix $i$ denotes the $i^{\text {th }}$ point and $\Delta x$ is the mesh spacing. The differencing of Equation (28) is equivalent to

$$
F_{X} \approx \frac{\partial}{\partial x}\left(F-\Delta x \varepsilon F_{X}\right)
$$

which is a conservation form of the equation. In the present method this form of differencing is used for the $\mathrm{F}_{\mathrm{x}}$ term and 
the values of $\phi_{X}$ in $F\left(\phi_{X}\right)$ are computed using central differencing. Otherwise the basic ITRAN2 algorithm is unchanged.

\section{DISCUSSION OF RESULTS}

As a first attempt at correcting the conventional unsteady small disturbance equation the correction was computed using Method I in which only the steady state solution is corrected. The first example is for the flow around a NACA 64A410 airfoil at $M_{\infty}=0.72$ and $\alpha=2^{\circ} \pm 2^{\circ} \sin k t$, where $k$ is the reduced frequency and is equal to 0.2 . The steady result is shown in figure 2 and it can be seen that the corrected result is a considerable improvement on the conventional TSD result. There is some additional discrepancy at the leading edge due to the inadequate treatment of the boundary condltions in the thin airfoil approximation.

In figure 3 the unsteady pressure distribution calculated by Option $I$ of the present method is shown for two stations on the oscillatory cycle. It can be seen that the present method gives too large a shock excursion and that the shock strength in the foremost position is much too weak. This is probably a consequence of the correction being only valid for the steady state solution. The conventional small disturbance solution procedure diverges rapidly in the unsteady mode due to excessive shock motion.

In figure 4 the steady pressure distributions around a NACA 0012 airfoil at $M_{\infty}=0.8$ and $\alpha=1.25^{\circ}$ for the present method, and the Euler equation solution of sells (ref. 8) is shown. It can be seen that the present results agree satisfactorily with the Euler equation solution as regards shock location but that the upper surface pressures are too high and the lower surface pressure is too low. However, the improvement over the conventional TSD result is substantial. The disagreement between potential 
equation results and Euler equation results in the leading edge region is also apparent in figure 4 if the results of Holst (ref. 9) are compared to the Euler equation results. Since TSD methods are usually "tuned" to approximate the full potential results it is possible that the discrepancy between the Euler solution and the present solution is due to fundamental differences in the numerical procedures used to solve full potential and Euler equations, since rotational effects should not be very important in the leading edge region.

In figure 5 the unsteady pressure distribution around a NACA 64 A410 airfoil at $m_{\infty}=0.72$ and $\alpha=2^{\circ} \pm 2^{\circ}$ sin $k t$ at $k=0.2$ is shown where Method II is used. It can be seen that this option considerably improves the shock behavior over that shown in figure 3 . It should be noted that part of the difference in results of the present method and the Euler equation method is due to the different mesh size and in the computations this effects the shock capture properties of the algorithms.

Finally in figure 6 a weak shock example is shown to illustrate the fact that the present method gives results close to the conventional TSD solution for weak shock waves. The airfoil is a NACA 0012 airfoil at $M_{\infty}=0.8$ and $\alpha= \pm 1 / 2^{\circ} \sin \mathrm{kt}$ with $\mathrm{k}=0.2$.

Although the present method does give substantially improved results over the conventional TSD solution, there are still several points regarding both the shock details in unsteady motion and the pressure distribution in regions of the flow outside the shock regions. It is desirable to obtain further Euler equation solutions for such an investigation. 


\section{CONCLUDING REMARKS}

A theory to correct the Transonic Small Disturbance equation to treat strong shock waves in unsteady flow has been developed. Although a fairly complete thoery has been developed, only a simplified form has been computationally implemented. The comparisons of results of the present method with solution of the Euler equations is adequate as regards the shock location but in certain cases the pressure distribution elsewhere on the airfoil surface is not satısfactory. It is suggested that the discrepancy may be due to inherent differences in the numerical scheme used to solve both sets of equations. Finally, although the present method gives a considerable improvement over the conventional TSD theory, it is desirable to further test the present theory in order to fully evaluate the technique.

\section{REFERENCES}

1. Murman, E. and Krupp, J. A.: Computation of Transonic Flows Past Lifting Airfoils and Slender Bodies. AIAA Journal, Vol. 10, No. 6, 1972, pp. 880-886.

2. Jameson, A.: Transonic Potential Flow Calculations Using Conservation Form. Proceedings AIAA 2nd Computational Dynamics Conference, 1975, pp. 148-161.

3. Magnus, R. and Yoshihara, H.: Calculations of Transonic Flow Over an Osclllating Airfoil. AIAA Journal, Vol. 13, No. 12, 1975, pp. 1622-1628.

4. Nixon, D.: Transonic Small Disturbance Theory with Strong Shock Waves. AIAA Journal, Vol. 18, No. 6, 1980, pp. 717-718.

5. Ballhaus, W. F. and Goorjian, P. F.: Implicit Finite Difference Computations of Unsteady Transonic Flows about Airfoils Including the Effects of Irregular Shock Motions. AIAA Journal, Vol. 15, Dec. 1977, pp. 1728-1735.

6. Ballhaus, W. V.: Some Recent Progress in Transonic Flow Computations. VKI Lecture Series: Computational Fluid Dynamics, von Karman Institute for Fluid Dynamics, 1976.

7. Liepmann, H. and Roshko, A.: Elements of Gas Dynamics. (Wiley), 1957. 


$$
-16-
$$

8. Sells, C. C. L.

9. Holst, T. 


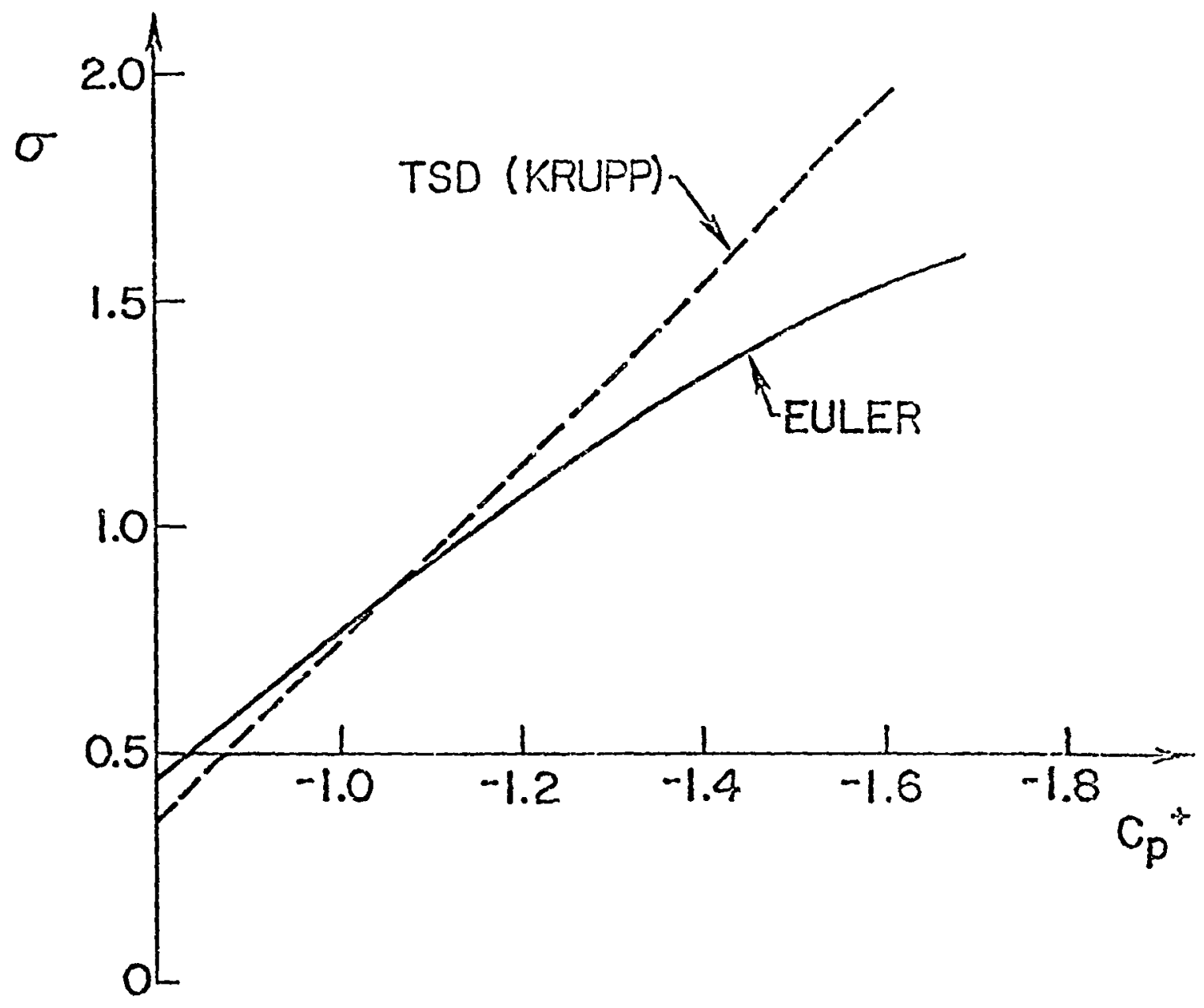

VARIATEONS OF NORHAL SHOCK STRENGTH WITH PRE-SHOCT PRFSSWRE

FIGURE I 


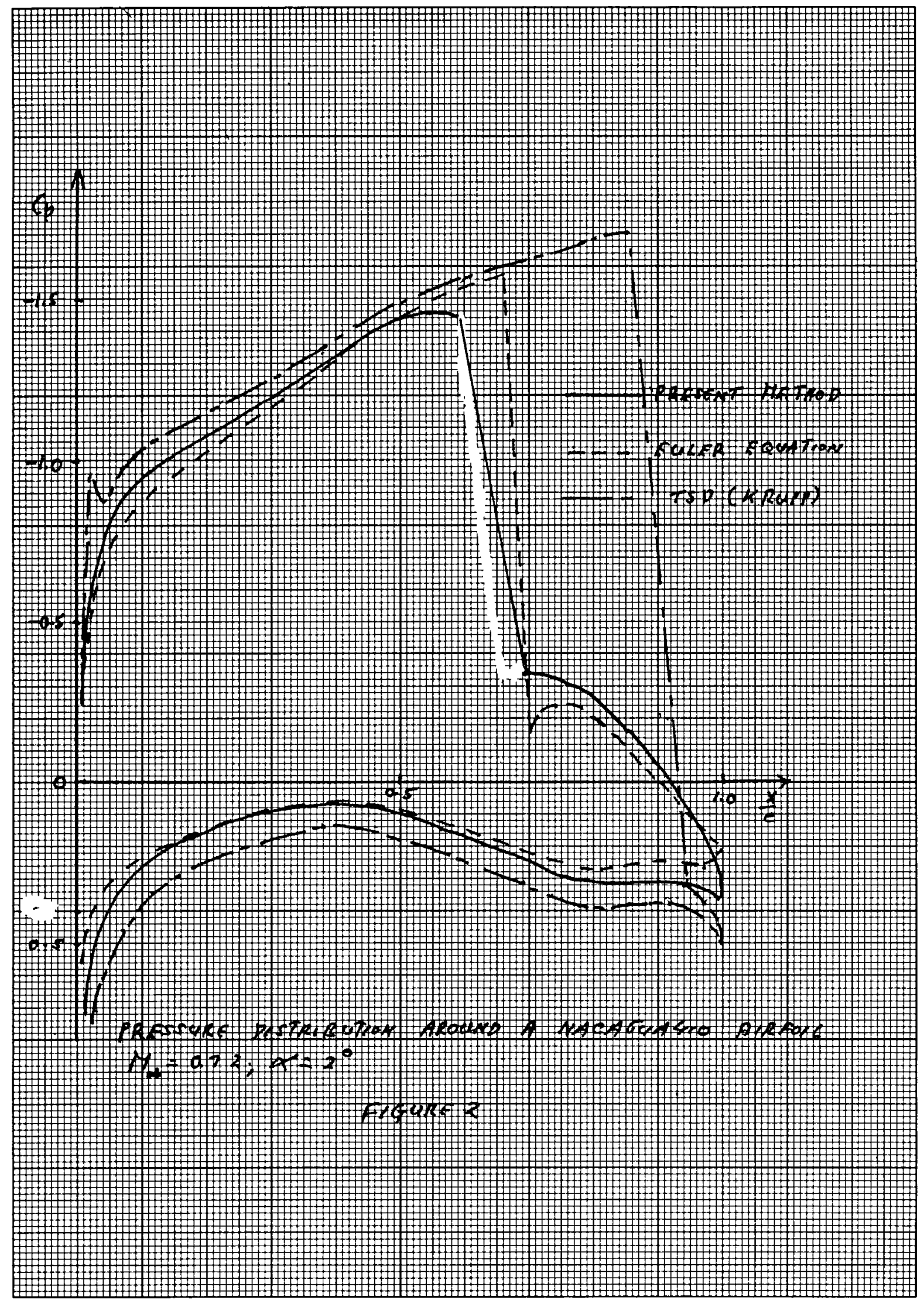




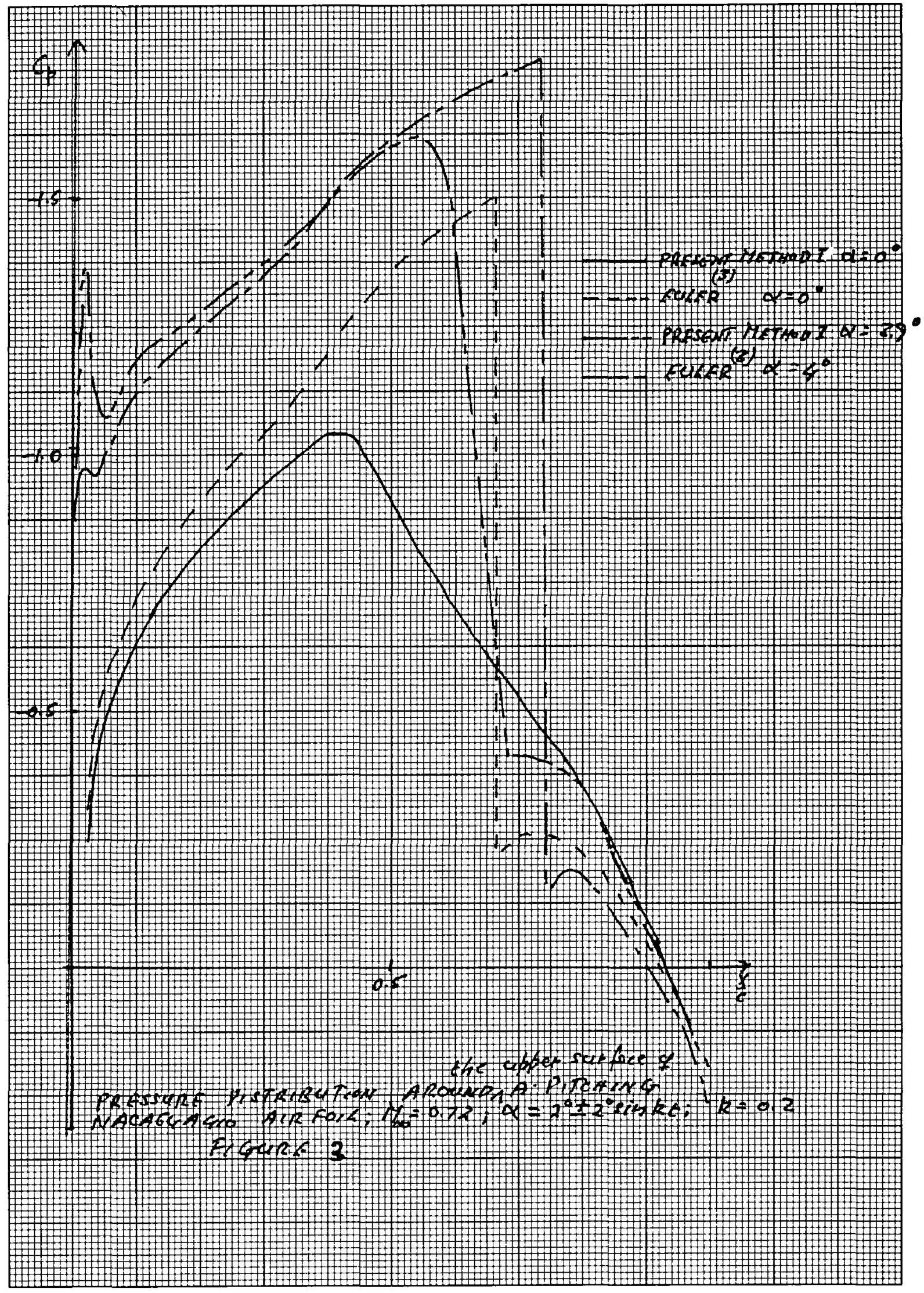




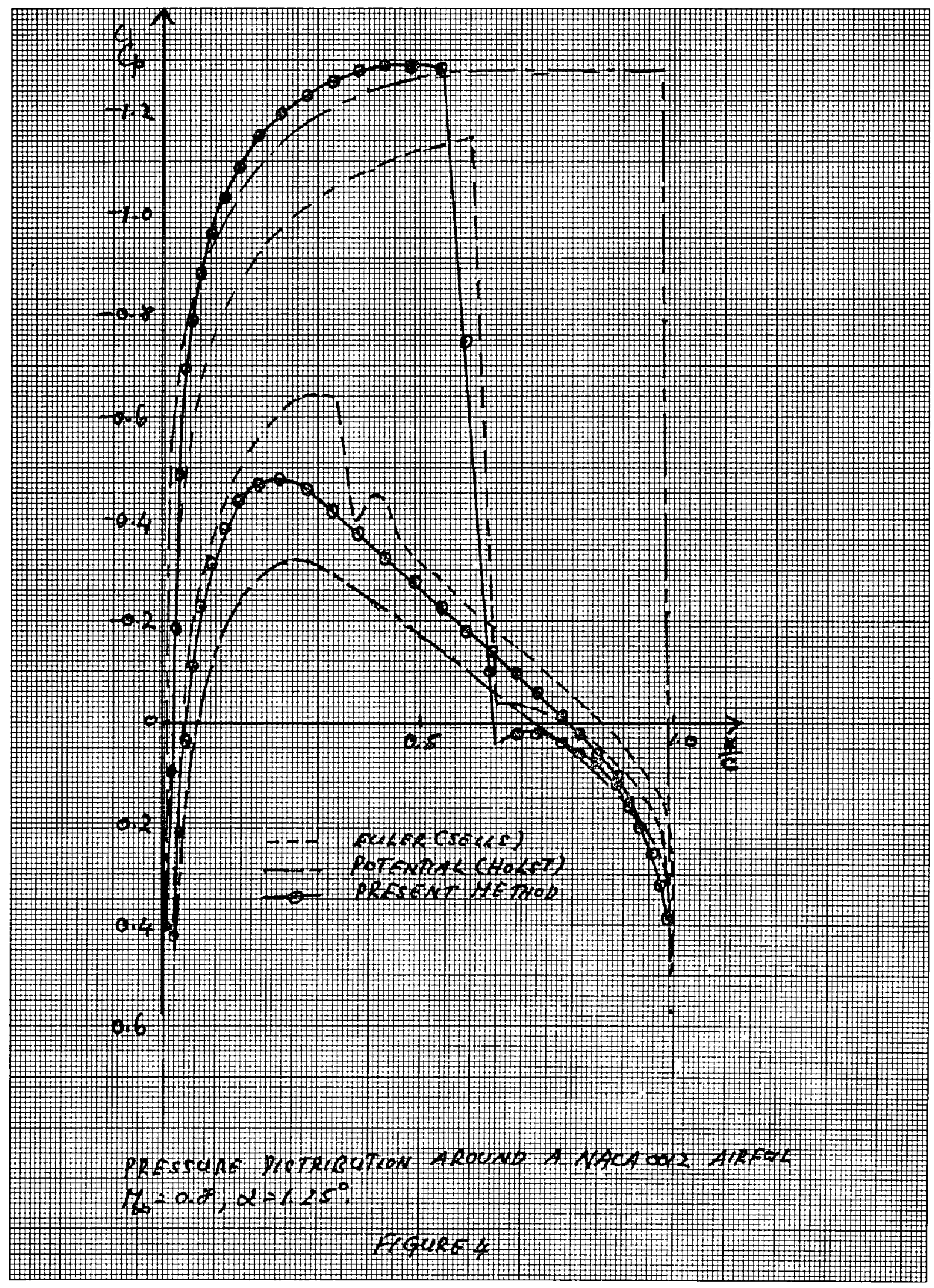




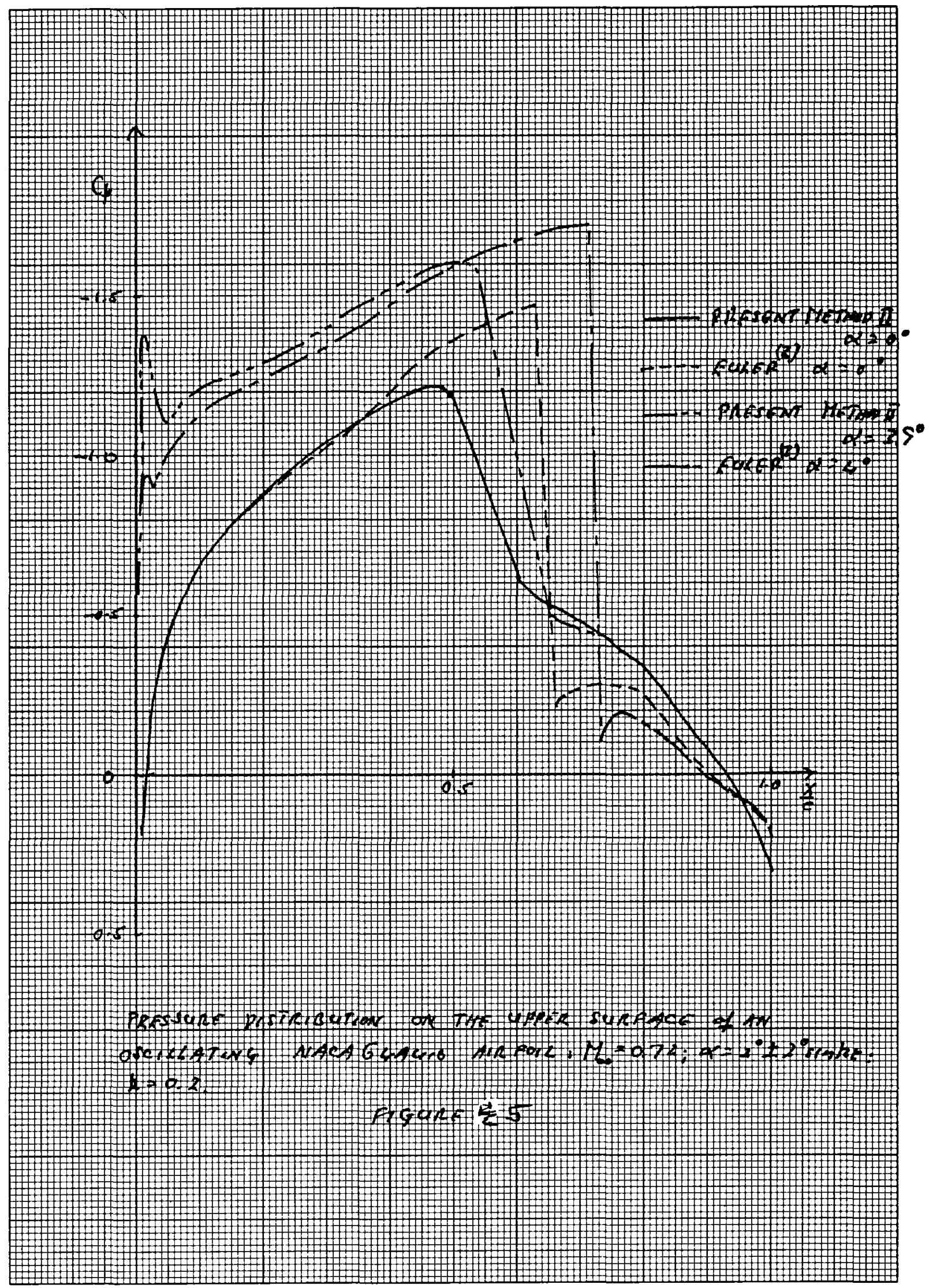




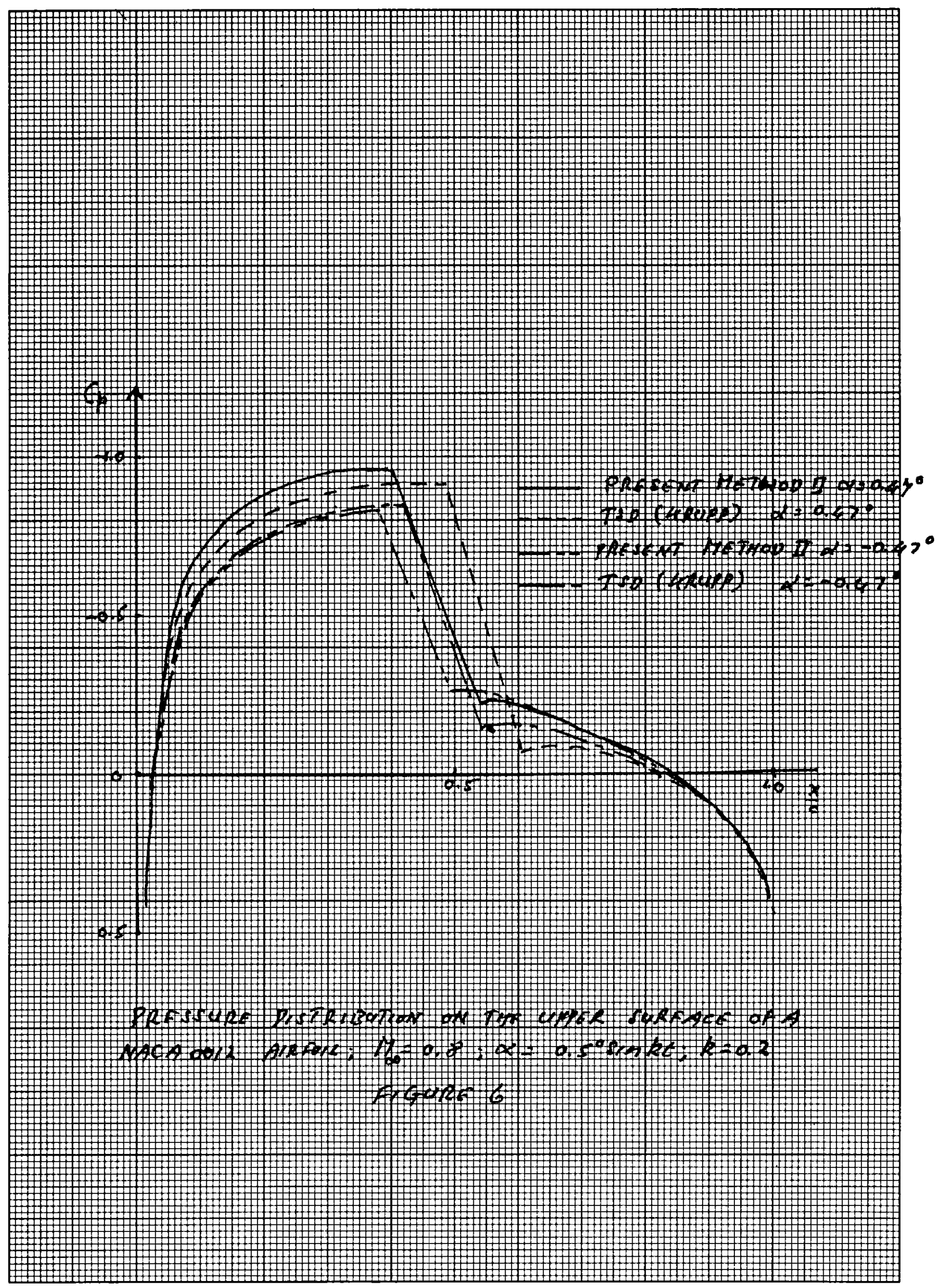


End of Document 